\section{P22 ARE WE USING THE BEST TESTS TO DIAGNOSE TV IN} GUM CLINICS IN THE UK?

${ }^{1}$ Jane Nicholls*, ${ }^{2}$ Peter Muir, ${ }^{3}$ Katy Turner, ${ }^{3}$ Margaret May, ${ }^{2}$ Paul North, ${ }^{3}$ John Macleod, ${ }^{3}$ Paddy Horner. 'Bristol Sexual Health Centre, Bristol, UK; ${ }^{2}$ Public Health England, Bristol Laboratory, Bristol, UK; ${ }^{3}$ Department of Social and Community Medicine, University of Bristol, Bristol, UK

\subsection{6/sextrans-2015-052126.66}

Background The Aptima TV NAAT test has been approved for use for the detection of Trichomonas vaginalis (TV) and is more sensitive $(\sim 100 \%)$ than wet mount microscopy $(50 \%)$ or culture (75\%). Asymptomatic women attending GUM clinics are often not tested for TV as the prevalence is assumed to be too low for testing to be cost effective.

Aims To determine

- TV positivity rate among GUM attendees with and without symptoms

- How many additional cases are identified with the new test

- Whether self-taken vaginal swabs are of equivalent sensitivity in symptomatic GUM patients.

Methods Patients were tested using the Aptima TV NAAT alongside existing testing methods. Test performance was compared using the McNemar test.

Results The positivity of TV determined by TV NAAT was $4.2 \%(22 / 519)$ in symptomatic and $1.8 \%(28 / 1599)$ in asymptomatic women. 9/20 NAAT positive patients, where all test were performed, would not have been identified on wet prep or culture. Overall TV NAAT outperformed currently used methods $(p=0.004)$, clinic wet prep vs NAAT $(p=0.038)$, culture vs NAAT $(p=0.002)$. Self-taken vaginal swabs were equivalent in sensitivity to clinician taken swabs; of patients who tested positive on either NAAT test, 19 tested positive on self-taken swab and 17 tested positive on clinician taken swab $(\mathrm{p}=0.625)$.

Conclusions Testing all women attending GUM clinics with the APTIMA TV NAAT test will identify additional cases and is therefore likely to be cost-effective, and should be considered to replace conventional microbiological testing methods.

\section{P23 INVESTIGATION OF THE ECONOMIC IMPACT OF IMPLEMENTING NATIONAL GUIDELINES TO RETEST YOUNG PEOPLE (AGED 16-24) WHO TEST POSITIVE FOR CHLAMYDIA}

${ }^{1}$ Katy Turner*, ${ }^{1}$ Katharine Looker, ${ }^{2}$ Georgina Angel, ${ }^{1,3}$ Paddy Horner, ${ }^{4}$ Sarah Woodhall, ${ }^{4}$ Kevin Dunbar, ${ }^{2}$ Norah O'Brien, ${ }^{5}$ Cecilia Priestley, ${ }^{3}$ Karl Pye, ${ }^{1}$ John Macleod, ${ }^{4}$ John Saunders. ${ }^{1}$ University of Bristol, Bristol, UK; ${ }^{2}$ Public Health England, Bristol, UK; ${ }^{3}$ University Hospital Bristol Trust, Bristol, UK; ${ }^{4}$ Public Health England, Colindale, UK; ${ }^{5}$ Park Centre for Sexual Health, Weymouth, UK

10.1136/sextrans-2015-052126.67

Background The National Chlamydia Screening Programme (NCSP) updated its guidelines in 2013 to recommend retesting for all chlamydia positive individuals around three months after treatment, due to the risk of reinfection.

Objectives Investigate the impact of implementing new retesting guidance on chlamydia screening activities and the economic cost of updating current testing practice.

Methods We developed a spreadsheet tool to calculate the additional costs of implementing new retesting guidance. We collected data from pilot evaluations of retesting to estimate the number of tests performed and the cost of administering retesting within existing services. We used these to estimate the national impact of the new guidelines, and to inform future updates to guidelines.

Results The baseline scenario is based on findings from pilot evaluations: for every 10,000 chlamydia tests, this will generate 750 positives (assuming 7.5\% positivity), of whom 40\% (300) would be retested within 6 months. This would identify an additional 30 positives $(10 \%$ positivity at retest). In this scenario, only $3 \%$ of all tests performed are retests, which would have minimal impact on the overall cost of the screening programme. The slight increased cost of retesting, associated with active recall of positive individuals is offset by the higher positivity observed at retest.

Conclusions The new guidelines to retest chlamydia positive individuals within 6 months appear feasible within the context of current programmes and will identify individuals at continued risk of infection with relatively low resource and time input.

\section{P24 OUTBREAK OR ILLUSION: CONSEQUENCES OF "IMPROVED" DIAGNOSTICS FOR GONORRHOEA}

${ }^{1}$ Amy Bennett*, ${ }^{1}$ Katie Jeffery, ${ }^{2}$ Eunan O'Neill, ${ }^{1}$ Jackie Sherrard. ${ }^{1}$ Oxford University Hospitals NHS Trust, Oxford, UK; ${ }^{2}$ Public Health Oxfordshire County Council, Oxford, UK

\subsection{6/sextrans-2015-052126.68}

Background/introduction The service introduced gonorrhoea nucleic acid testing (NAATs) using the BD Viper LT $^{\mathrm{TM}}$ System in August 2012. Since then rates of gonorrhoea have increased threefold (Table 1). Concerns were raised by Public Health England in 2014 that this increase represented an outbreak.

\begin{tabular}{llll}
\multicolumn{5}{l}{ Abstract P24 Table 1} & Gonorrhoea rates \\
\hline & $\%$ all males & $\%$ MSM & $\%$ all females \\
\hline July-Dec 2011 & $40 / 4789(0.8 \%)$ & $11 / 283(3.9 \%)$ & $7 / 5546(0.2 \%)$ \\
Jan-June 2012 & $43 / 4783(0.9 \%)$ & $15 / 249(6 \%)$ & $18 / 5474(0.3 \%)$ \\
July-Dec 2012 & $89 / 5002(1.8 \%)$ & $50 / 377(13.2 \%)$ & $17 / 5499(0.3 \%)$ \\
Jan-June 2013 & $94 / 4957(1.9 \%)$ & $60 / 525(11.4 \%)$ & $25 / 5445(0.4 \%)$ \\
July-Dec 2013 & $102 / 4838(2.0 \%)$ & $63 / 557(11.3 \%)$ & $42 / 5702(0.7 \%)$ \\
Jan-June 2014 & $115 / 5221(2.2 \%)$ & N/A & $53 / 5936(0.8 \%)$ \\
\hline
\end{tabular}

Aim(s)/objectives To ascertain if there was an outbreak.

Methods We reviewed all 153 gonorrhoea (GC) cases seen from January to June 2014.

Results Of 45 female cases, 16 (36\%) were not known GC contacts, and were culture negative: all were NAATS positive at the cervix. Of 43 cases in heterosexual men, 4 were positive by NAATs only and not known contacts of GC: one had a single partner who tested negative for GC. There were 65 cases in MSM. Of 36 (55\%) NAATS positive only who were asymptomatic and not a known GC contact, 32 had isolated pharyngeal infection, 3 rectal infections only and 1 dual rectal and pharynx infection.

Discussion/conclusion At an incident control meeting with the local authority, PHE and local GUM service, it was agreed there was insufficient evidence to confirm a cluster of cases and that at least some of the increase could be attributable to the introduction of NAATs testing. It was agreed to prospectively audit GC cases, until March 2015 and to send NAAT positive/culture negative samples to reference laboratory for confirmatory testing. Initial results from the first 2 months suggest that a significant number of cases are not confirmed. The full data will be 